\section{(2) OPEN ACCESS}

- Additional material is published online only. To view please visit the journal online (http://dx.doi.org/10.1136/ thoraxjnl-2019-214317).

For numbered affiliations see end of article.

\section{Correspondence to} Eric C J Claas, Medical Microbiology, Leiden Universitair Medisch Centrum, Leiden 2300RC, The Netherlands; E.C.J.Claas@lumc.nl

KTZ and ALVR contributed equally.

Received 12 November 2019 Revised 14 April 2020 Accepted 18 June 2020 Published Online First 20 August 2020

\title{
Molecular epidemiology and clinical impact of rhinovirus infections in adults during three epidemic seasons in 11 European countries (2007-2010)
}

\author{
Kalina T Zlateva, ${ }^{1}$ Anneloes L van Rijn (1) , ${ }^{1}$ Peter Simmonds, ${ }^{2}$ Frank E J Coenjaerts, ${ }^{3}$ \\ Anton M van Loon, ${ }^{3}$ Theo J M Verheij, ${ }^{4}$ Jutte J C de Vries, ${ }^{1}$ Paul Little (ㄱ, ${ }^{5}$ \\ Christopher C Butler, ${ }^{6}$ Erik W van Zwet, ${ }_{1}^{7}$ Herman Goossens, ${ }^{8}$ Margareta leven, ${ }^{8}$ \\ Eric C J Claas, ${ }^{1}$ on behalf of the GRACE Study Group
}

\begin{abstract}
Background Differences in clinical impact between rhinovirus (RVs) species and types in adults are not well established. The objective of this study was to determine the epidemiology and clinical impact of the different RV species.

Methods We conducted a prospective study of RVs infections in adults with acute cough/lower respiratory tract infection (LRTI) and asymptomatic controls. Subjects were recruited from 16 primary care networks located in 11 European countries between 2007 and 2010. RV detection and genotyping was performed by means of real time and conventional reverse-transcriptase polymerase chain reaction assays, followed by sequence analysis. Clinical data were obtained from medical records and patient symptom diaries.

Results RVs were detected in 566 (19\%) of 3016 symptomatic adults, 102 (4\%) of their 2539 follow-up samples and 67 (4\%) of 1677 asymptomatic controls.

\section{Key messages}

What is the key question?

- What is the epidemiology and clinical impact of the different rhinovirus (RV) species.

What is the bottom line?

- RV-A was significantly more often detected in adults with acute lower respiratory tract infections than RV-C, while RV-B infection was often found in asymptomatic patients.

Why read on?

- The epidemiology and clinical impact of RVs is poorly understood and this is the most comprehensive assessment of RV speciesspecific clinical outcomes in adults with symptoms of lower respiratory tract infection visiting their general practitioner.
\end{abstract} Genotyping was successful for 538 (95\%) symptomatic subjects, 86 (84\%) follow-up infections and 62 (93\%) controls. RV-A was the prevailing species, associated with an increased risk of LRTI as compared with RV-B (relative risk (RR), 4.5; 95\% Cl 2.5 to $7.9 ; p<0.001$ ) and RV-C (RR 2.2; 95\% Cl 1.2 to 3.9; $p=0.010$ ). In symptomatic subjects, RV-A loads were higher than those of RV-B $(p=0.015)$. Symptom scores and duration were similar across species. More RV-A infected patients felt generally unwell in comparison to RV-C $(p=0 \cdot 023)$. Of the 140 RV types identified, five were new types; asymptomatic infections were associated with multiple types. Interpretation In adults, RV-A is significantly more often detected in cases with acute cough/LRTI than RV$C$, while RV-B infection is often found in asymptomatic patients.

\section{INTRODUCTION}

Rhinoviruses (RVs) are the most prevalent respiratory pathogens in humans, accounting for approximately $20 \%-50 \%$ of respiratory tract infections (RTIs) annually. ${ }^{1-4}$ Infection with RV can lead to a wide spectrum of clinical manifestations ranging from asymptomatic to severe lower respiratory illness. ${ }^{256} \mathrm{RV}$ is also recognised as a major cause of exacerbations of chronic obstructive pulmonary disease (COPD), asthma and cystic fibrosis. ${ }^{78}$ Coinfections with other viral or bacterial pathogens have been frequently reported, potentially increasing clinical severity. ${ }^{5} 910$ Over 160 RV types have been identified and classified into three genetically distinct species (RV-A, RV-B and $\mathrm{RV}-\mathrm{C}$ ) within the genus Enterovirus of the family Picornaviridae. ${ }^{11} \mathrm{RV}$-A has been the most frequently detected RV species, followed by RV-C and RV-B, respectively. ${ }^{34}$ Although the clinical significance of RV species and types remains poorly defined, RV-C has been predominantly linked to asthma exacerbations and more serious respiratory illness in young children. ${ }^{12-14}$ More recently similar clinical presentations across species have been reported. ${ }^{15-18}$

Despite the high morbidity and disease burden of RV infections, there are currently no effective and safe antiviral treatments, and efforts to develop an effective vaccine have been hampered by the substantial antigenic diversity between RV types and species. A more practical approach could be the development of vaccines targeting more prevalent or pathogenic RV types although the feasibility of this depends on whether particular RV species or types are associated with more frequent or severe respiratory illness. Previous studies have been limited by the absence of asymptomatic controls 
and incomplete microbiological investigation, and were focused mainly on hospitalised young children.

The aim of this study was to determine the epidemiology and clinical impact of the different RV species through a, communitybased comparative evaluation of disease severity among adult subjects infected with different RV species and types. Additionally, the multicentre approach provides insight in geographical differences in RV epidemiology.

\section{METHODS}

\section{Study design and subjects}

Respiratory samples were obtained from subjects aged 18 years and older enrolled as part of the genomics to combat resistance against antibiotics community-acquired lower respiratory tract infections (LRTIs) in Europe (GRACE) European Network of Excellence (www.grace-lrti.org) focusing on improving the management of community-acquired LRTIs. Sixteen primary care networks from 11 European countries including: Belgium (Antwerp and Ghent); France (Nice), Germany (Rotenberg), Italy (Milan), the Netherlands (Utrecht), Poland (Bialystok, Lodz and Szczecin), Slovakia (Bratislava), Slovenia (Jesenice), Spain (Barcelona and Mataró), Sweden (Jönköping) and UK (Cardiff and Southampton) participated in prospective recruitment of patients presenting with acute cough and signs of an acute LRTI over the period October 2007-June 2010. Cases were patients presenting at the general practitioner (GP) with an acute or worsened cough ( $\leq 28$ days duration) as the main symptom, or any clinical presentation considered by the GP to be caused by LRTI and consulting for the first time for this illness episode. Exclusion criteria were pregnancy, breast feeding and any condition associated with severe impaired immune status. Asymptomatic controls were subjects who consulted their GP for complaints other than respiratory, who had no symptoms and signs of a RTI and had not used antibiotics or antivirals in the previous 2 weeks. Case and controls were recruited from the same GP centre, within 14 days of each other and with a maximum 5-year age difference.

Nasopharyngeal swab (NPS) and sputum (if available) samples were collected from case (V1) and control (C1) patients during their first visit to the GP, for which standardisation was obtained by training of staff. Follow-up NPS samples were obtained from case patients at the second visit (V2) to the GP after approximately 28 days. Medical ethics committees in participating countries approved the study and all participants provided written informed consent.

The original study was designed to evaluate the aetiology of LRTIs in GP settings, as described by Ieven et al, this study is a post hoc analysis. ${ }^{19}$

\section{Data collection}

Clinical data, including medical history, comorbidities and their management/treatment, and days the patient felt unwell were recorded by the GP on a case report form (CRF) at the first consultation. The presence or absence of cough, sputum production, shortness of breath, wheeze, coryza, fever, chest pain, muscle aching, headache, disturbed sleep, feeling generally unwell, interference with normal activities, confusion/ disorientation and diarrhoea, were documented. If present, the severity of each symptom was rated on a 4-point scale ranging from 'no problem $=1$ ' to 'severe problem $=4$ '. Following the first GP consultation, patients were requested to complete a daily symptom diary for the duration of illness (to a maximum of 28 days). The presence and severity of the above symptoms (except diarrhoea) were rated on a 7-point scale ranging from 'normal/ not affected $=0$ ' to ' as bad as it could be $=6$ '.

\section{Clinical assessment}

The disease severity of RV infections was evaluated by comparing the following clinical characteristics: (1) initial symptom score, (2) duration of illness, (3) maximal symptom score and (4) duration of higher symptom score. The 'initial symptom score' was estimated as the mean severity score of at least 12 of the 14 symptoms evaluated by the GP during the first visit of the patient. The 'duration of illness', 'maximal symptom score' and 'duration of higher symptom score' were estimated based on the presence and severity of 13 symptoms excluding diarrhoea, recorded in the patient's daily symptom diary as described previously. The duration of illness was defined as the total sum of days a patient felt unwell before the first GP visit and the number of days the patient experienced any of the 13 symptoms over the 28 days follow-up period. The duration of higher symptom score was the number of days a patient had a mean daily symptom score of two or more based on the 7 -point scale. ${ }^{20}$ The occurrence and severity of each symptom recorded during the first visit to the GP were also compared between RV species.

\section{Laboratory investigation}

The overall results of the laboratory diagnostics were previously described by Ieven et al. ${ }^{19}$ In short, total nucleic acids were extracted at the University Hospital Antwerp, and examined for RV by real-time PCR (RT-PCR) at the Leiden University Medical Center. Cycle quantification (Cq) values, which had been normalised using a fixed baseline fluorescence threshold, were used as an indicative measure of viral load. Bacterial and fungal infections were detected in NPS or/and sputum samples by conventional culture and/or molecular methods. ${ }^{21}$

\section{RV typing}

RV genotyping was conducted by amplification and sequencing of partial VP3/VP1, VP4/VP2 or/and 5' - untranslated region (5'-UTR) viral genome fragments as described previously. ${ }^{20}$ Basic local alignment search tool with nucleotide database (BLASTn) analyses were conducted for initial species identification and then confirmed by phylogenetic analysis. RV sequences were compared with prototype strains and assigned to types based on phylogenetic analysis and pairwise $\mathrm{p}$ distances (see online supplementary appendix). RV sequences from this study have been deposited in GenBank under the accession numbers KP736530-KP737279 and KR045604.

\section{Statistical analysis}

Continuous variables were summarised as medians with IQR ranges or means with $95 \% \mathrm{CI}$ and for categorical variables, the frequencies in each category were calculated. Demographic and clinical characteristics were compared across RV species within RV-positive symptomatic and asymptomatic adults by using risk ratio and/or $\mathrm{X}^{2}$ tests for categorical variables and the non-parametric Kruskal-Wallis test, Mann-Whitney $U$ test or independent t-test or one-way analysis of variance when appropriate for continuous variables. Correction for possible confounders (age, sex, geographical location, sample season and sample year) was performed using multivariable linear or logistic regression for continuous or binary outcome measures, respectively. The statistical analyses were conducted in the IBM SPSS Statistics software V.25 for Windows (IBM). A two sided $\mathrm{p}<0 \cdot 05$ was considered statistically significant. 

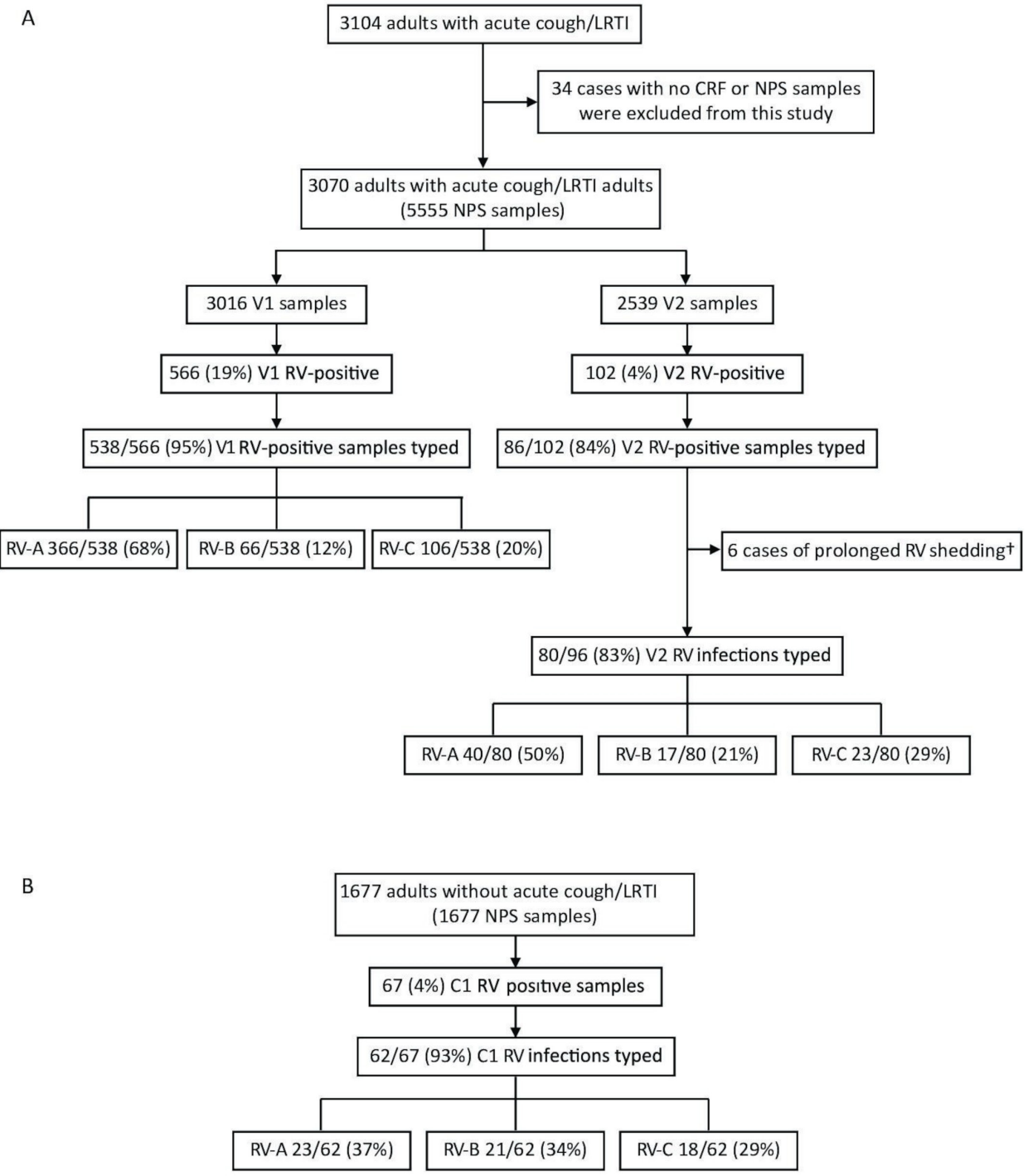

Figure 1 Rhinovirus prevalence and species distribution among (A) adults enrolled with acute cough/ LRTI and asymptomatic (B) controls. CRF, case report form; LRTI, lower respiratory tract infection; NPS, nasopharyngeal swab; RV, rhinovirus. †Prolonged rhinovirus shedding was identified in 6 patients.

\section{RESULTS}

\section{RV detection and species distribution}

In this study, 3070 (99\%) (median age 50, (IQR 36-63 years); male/female ratio $1: 1 \cdot 5)$ of 3104 recruited adults with acute cough/LRTI and 1677 asymptomatic controls (median age 50, (IQR 35-62 years); male/female ratio 1:1.5) were included. Thirty-four patients with no available NPS material or CRF were excluded (figure 1). NPS samples were obtained from 3016 symptomatic patients during the first visit (V1) to the GP and a follow-up (V2) sample was collected from 2485 (82\%) of these patients. ${ }^{20}$ For 54 symptomatic patients, only a follow-up NPS was available for testing, resulting in a total of 2539 V2 NPS specimens. Of the asymptomatic controls 1677 samples were tested. Of the 3104 symptomatic patients 1844 (60\%) tested positive for a respiratory pathogen, of which 350 (11\%) a bacterial pathogen only, 1190 (39\%) a respiratory virus and 304 (10\%) a double infection. Of the 1677 asymptomatic controls, $205(12 \%)$ had an respiratory virus as previously reported by Ieven et al. ${ }^{19}$

RV was detected in 766 out of 7232 samples (11\%) and genotyping was successful for 717 (94\%) of these samples.
Enteroviruses, including 13 EV-D68, 10 EV-C104, 5 EV-C105, $2 \mathrm{EV}-\mathrm{C} 117$ types and $1 \mathrm{EV}-\mathrm{C}$ (non-typeable), were identified due to cross-reactivity in our diagnostic RV assay. These $31 \mathrm{EV}$-positive samples were excluded from further analysis. Forty-nine (6\%) RV-positive samples with lower viral loads inferred from quantification cycle $(\mathrm{Cq})$ values could not be genotyped due to amplification failure in the genotyping PCR (median Cq values of 29 vs 36 in the diagnostic RT-PCR assay for genotyped and not-genotyped samples, respectively; $\mathrm{p}<0.001)$.

RV was detected in 566 (19\%) V1 and 102 (4\%) V2 samples from case patients (of which 21 were positive in both the V1 and V2 samples) and in 67 (4\%) C1 samples from asymptomatic control patients. ${ }^{20}$ Of the $735 \mathrm{RV}$ infection episodes, 182 (25\%) were coinfections, of which 68 (9\%) with another virus, $103(14 \%)$ with a bacterial coinfection and $11(2 \%)$ had an RV infection with at least two other pathogens. Commensals and Candida species were not considered causative pathogens and were excluded from the analysis.

Symptomatic (V1 positive) RV-positive patients tended to be older than asymptomatic patients (median 47 years, (IQR 33-60 years) vs median 38 years (IQR 27-58 years); $\mathrm{p}=0 \cdot 058$, 
Table 1 Demographics of symptomatic (V1) vs asymptomatic (C1) RV-positive patients

\begin{tabular}{|c|c|c|c|c|}
\hline & $\begin{array}{l}\text { Total } \\
n=633\end{array}$ & $\begin{array}{l}R V+V 1 \\
n=566\end{array}$ & $\begin{array}{l}\mathrm{RV}+\mathrm{C} 1 \\
\mathrm{n}=67\end{array}$ & $P$ value \\
\hline Male (\%) & $263(42)$ & $229(40)$ & $34(51)$ & 0.106 \\
\hline Age (median, IQR) & $47(32-60)$ & $47(33-60)$ & $38(27-58)$ & 0.058 \\
\hline Ethical background (\%) & 563 & & & \\
\hline Caucasian & & $540(96)$ & & \\
\hline African & & $8(1)$ & & \\
\hline Asian & & $10(2)$ & & \\
\hline Other & & $5(1)$ & & \\
\hline Study site (\%) & & & & 0.281 \\
\hline Antwerp BE & $95(15)$ & $85(15)$ & $10(15)$ & \\
\hline Barcelona SP & $66(10)$ & $64(11)$ & $2(3)$ & \\
\hline Bialystok PL & $28(4)$ & $24(4)$ & $4(6)$ & \\
\hline Bratislava SL & $30(5)$ & $29(5)$ & $1(1)$ & \\
\hline Cardiff GB & $64(10)$ & $57(10)$ & $7(10)$ & \\
\hline Ghent BE & $25(4)$ & $20(4)$ & $5(7)$ & \\
\hline Jesenice SVN & $10(2)$ & $10(2)$ & $0(0)$ & \\
\hline Jonkoping SW & $8(1)$ & $7(1)$ & $1(1)$ & \\
\hline Lodz PL & $52(8)$ & $47(8)$ & $5(7)$ & \\
\hline Mataró SP & $79(12)$ & $68(12)$ & $11(16)$ & \\
\hline Milan IT & $7(3)$ & $17(3)$ & $0(0)$ & \\
\hline Nice FR & $3(0.5)$ & $3(1)$ & $0(0)$ & \\
\hline Rotenberg DU & $27(4)$ & $25(4)$ & $2(3)$ & \\
\hline Southampton GB & $43(7)$ & $39(7)$ & $4(6)$ & \\
\hline Szczecin PL & $27(4)$ & $22(4)$ & $5(7)$ & \\
\hline Utrecht NL & $59(9)$ & $49(9)$ & $10(15)$ & \\
\hline Sample season and year (\%) & 632 & 565 & 67 & $0.918^{*}$ \\
\hline Autumn 07 & $37(6)$ & $34(6)$ & $3(4)$ & \\
\hline Winter 07/08 & $49(8)$ & $43(8)$ & $6(9)$ & \\
\hline Spring 08 & $73(12)$ & $67(12)$ & $6(9)$ & \\
\hline Summer 08 & $4(1)$ & $4(1)$ & $0(0)$ & \\
\hline Autumn 08 & $118(19)$ & $106(19)$ & $12(18)$ & \\
\hline Winter 08/09 & $64(10)$ & $55(10)$ & $9(13)$ & \\
\hline Spring 09 & $44(7)$ & $40(7)$ & $4(6)$ & \\
\hline Summer 09 & $4(1)$ & $4(1)$ & $0(0)$ & \\
\hline Autumn 09 & $131(37)$ & $118(21)$ & $13(19)$ & \\
\hline Winter 09/10 & $77(12)$ & $69(12)$ & $8(12)$ & \\
\hline Spring 10 & $31(5)$ & $25(4)$ & $6(9)$ & \\
\hline \multicolumn{2}{|l|}{ Underlying condition (\%) } & \multicolumn{3}{|l|}{$238 / 563(42)$} \\
\hline \multicolumn{2}{|l|}{ Asthma } & \multicolumn{3}{|l|}{$67 / 563(12)$} \\
\hline \multicolumn{2}{|l|}{ COPD } & \multicolumn{3}{|l|}{$41 / 563(7)$} \\
\hline \multicolumn{2}{|c|}{ Other respiratory comorbidities } & \multicolumn{3}{|l|}{$7 / 563$ (1) } \\
\hline \multicolumn{2}{|c|}{$\begin{array}{l}\text { History of hospitalisation for respiratory } \\
\text { illness }\end{array}$} & \multicolumn{3}{|l|}{$24 / 563(4)$} \\
\hline \multicolumn{2}{|l|}{ Cardiac disease } & \multicolumn{3}{|l|}{$47 / 563(8)$} \\
\hline \multicolumn{2}{|l|}{ Diabetes } & \multicolumn{3}{|l|}{$35 / 562(6)$} \\
\hline \multicolumn{2}{|l|}{ Allergic diseases } & \multicolumn{3}{|l|}{$105 / 563(23)$} \\
\hline
\end{tabular}

respectively), see table 1 . No significant differences were found in sex, location and sample season and year between RV-positive symptomatic and asymptomatic adults. Of 563 of the $566 \mathrm{~V} 1$ patients underlying conditions were stated: 238 (42\%) had an underlying condition, including 122 (51\%) with respiratory comorbidities or history of hospitalisation for respiratory illness. Mixed infections within the RV-positive adults (V1) were associated with symptomatic disease more often (29\% vs $7 \%$ in the asymptomatic patients, RR 4.9 (95\% CI 2.0 to 11.8$) \mathrm{p}<0.001$, adjusted for age, sex, geographical location, sample season, sample year and viral load). Mixed infections were not included in further analyses. The RV-viral load was lower in the RV-positive asymptomatic controls (mean Cq value 31 vs 28 in patients, $\mathrm{p}<0.001$, adjusted for age, sex, geographical location, sample season, sample year and mixed infections).

Virus genotyping to RV species level was successful in 538 (95\%) V1, 86 (84\%) V2 RV-positive samples from symptomatic patients and in 62 (93\%) C1 control subjects. The number and proportion of RV species among symptomatic, follow-up and asymptomatic infections are shown in figure 1 . Prolonged RV shedding in symptomatic patients (V1) was identified in six patients as previously described. ${ }^{20} \mathrm{RV}$ reinfection with another RV type was identified in 11 symptomatic (V1) patients.

\section{Clinical manifestations}

Complete clinical record forms and symptom diaries were available for 563 (99.5\%) and 383 (67,7\%) RV-positive symptomatic V1 patients, respectively. Age and sex characteristics of symptomatic and asymptomatic subjects, infection outcome, viral loads inferred from Cq values and duration and severity of disease were compared across RV species (table 2). The age and sex distribution was similar across RV-species in the symptomatic and control groups. In $538 \mathrm{RV}$-infected symptomatic patients the proportion of RV-A infections was $68 \%$ vs $12 \%$ for RV-B and $20 \%$ for RV-C infections. In contrast, in the 62 asymptomatic infections the proportions were similar: RV-A: 37\%, RV-B: 34\% and RV-C: 29\% (figure 1). For single infections RV-A was 4.5 and 2.2 times more often associated with a LRTI outcome than RV-B $(p<0 \cdot 001)$ and RV-C $(p=0 \cdot 010)$, respectively, and RV-C more often than RV-B (RR 2.1, $\mathrm{p}=0.015)$. The results were similar for RV coinfected cases. In symptomatic patients with a RV monoinfection RV-A viral loads (mean $\mathrm{Cq}=27$ ) were significantly higher than RV-B viral loads (mean $\mathrm{Cq}=30, \mathrm{p}=0.015$ ). The duration, overall disease severity, and maximum daily symptom score were similar between RV species. The severity of the individual symptoms was also compared across RV species (table 3). Significant differences were observed only for 'feeling generally unwell'. In particular RV-A infected patients generally felt worse than those with RV-C ( $p=0.023)$. No other significant differences were observed between RV-A and RV-B, or RV-C and $\mathrm{RV}-\mathrm{B}$ infected patients.

\section{RV prevalence and species distribution according to site and year of detection}

The number of tested NPS samples, RV detection rate and species distribution among the 16 sites in Europe over the 3 years study period are presented in figure 2 . The majority of subjects were recruited in the months October to April and years were defined from July until June of the following year. No patients were recruited from Nice, Bialystock, Szczecin, Bratislava and Jesenice during 2007/2008 and only 9 samples from six patients were available from Milan for 2009/2010. RV accounted for 1\% up to $21 \%$ of respiratory infections per site during a particular year, when excluding Milan during 2009/2010, because of the low number of included cases. 
Table 2 Age and sex distribution, infection outcome and disease severity according to rhinovirus species

\begin{tabular}{|c|c|c|c|c|c|c|}
\hline Characteristics* & RV-A & RV-B & RV-C & $\mathrm{RR}(95 \% \mathrm{Cl})$ & \multicolumn{2}{|l|}{$P$ value } \\
\hline \multicolumn{7}{|l|}{ Age/sex -V1 infections } \\
\hline No of cases & 366 & 66 & 106 & & & \\
\hline Median (IQR) years & $47(33-60)$ & $43(30-61)$ & $50(33-62)$ & & \multicolumn{2}{|l|}{0.571} \\
\hline No $(\%)$ of male & $155(42)$ & $22(33)$ & $43(41)$ & & \multicolumn{2}{|l|}{0.389} \\
\hline \multicolumn{7}{|l|}{ Age/sex -C1 infections } \\
\hline No of cases & 23 & 21 & 18 & & & \\
\hline Median (IQR) years & $37(25-53)$ & $36(28-51)$ & $50(30-61)$ & & \multicolumn{2}{|l|}{0.472} \\
\hline No $(\%)$ of male & $13(57)$ & $9(43)$ & $10(56)$ & & \multicolumn{2}{|l|}{0.613} \\
\hline $\begin{array}{l}\text { Symptomatic (V1) vs asymptomatic } \\
\text { (C1) infections }\end{array}$ & $366(94) / 23(6)$ & $66(76) / 21(24)$ & $106(85) / 18(15)$ & RV-A vs RV-B: RR 4.6 (2.7 to 8.0 ) & \multicolumn{2}{|c|}{ RV-A vs RV-B $(<0.001) \dagger$} \\
\hline \multirow[t]{2}{*}{ No (\%) of V1/C1 cases } & & & & RV-A vs RV-C: RR 2.4 (1.4 to 4.3 ) & \multicolumn{2}{|c|}{ RV-A vs RV-C (0.003)† } \\
\hline & & & & RV-C vs RV-B: RR 1.9 (1.1 to 3.4) & \multicolumn{2}{|c|}{ RV-C vs RV-B (0.026) $\dagger$} \\
\hline $\begin{array}{l}\text { Symptomatic (V1) vs asymptomatic } \\
\text { (C1) single infections }\end{array}$ & $261(92) / 22(8)$ & $43(70) / 18(32)$ & $80(82) / 17(18)$ & RV-A vs RV-B: RR 4.5 (2.5 to 7.9 ) & \multicolumn{2}{|c|}{ RV-A vs RV-B $(<0.001) \dagger$} \\
\hline \multirow[t]{2}{*}{ No $(\%)$ of $\mathrm{V} 1 / \mathrm{C} 1$ cases } & & & & RV-A vs RV-C: RR 2.2 (1.2 to 3.9) & \multicolumn{2}{|c|}{ RV-A vs RV-C $(0.010) \dagger$} \\
\hline & & & & RV-C vs RV-B: RR 2.1 (1.1 to 3.7) & \multicolumn{2}{|c|}{ RV-C vs RV-B (0.015) $\dagger$} \\
\hline Cq values- $\mathrm{V} 1$ single infections & 27 (27 to 28$)$ & 30 (28 to 31$)$ & 27 (26 to 29$)$ & & 0.053 & RV-A vs RV-B $(0.015) \dagger$ \\
\hline \multirow[t]{2}{*}{ Mean $(95 \% \mathrm{Cl})$} & & & & & & RV-A vs RV-C (0.780)t \\
\hline & & & & & & RV-C vs RV-B (0.068) $\dagger$ \\
\hline Cq values- $\mathrm{C} 1$ single infections & 31 (29 to 33 ) & 32 (30 to 33 ) & 31 (29 to 32 ) & & $0.838 \dagger$ & RV-A vs RV-B (0.922) \\
\hline \multirow[t]{2}{*}{ Mean $(95 \% \mathrm{Cl})$} & & & & & & RV-A vs RV-C (0.576) $\dagger$ \\
\hline & & & & & & RV-C vs RV-B (0.642) $\dagger$ \\
\hline Initial symptom scoreł & & & & & $0.697 \dagger$ & RV-A vs RV-B (0.426) $\dagger$ \\
\hline No of cases & 260 & 43 & 80 & & & RV-A vs RV-C (0.666) $\dagger$ \\
\hline Median (IQR) & $1.6(1.1-2.0)$ & $1.4(0.9-2.1)$ & $1.4(1.1-1.9)$ & & & RV-C vs RV-B $(0.684) \dagger$ \\
\hline Duration of illness & & & & & $0.987 \dagger$ & RV-A vs RV-B (0.893) $\dagger$ \\
\hline No of cases & 200 & 31 & 62 & & & RV-A vs RV-C (0.948) $\dagger$ \\
\hline Median (IQR) days & $21(14-31)$ & $20(13-31)$ & $19(13-32)$ & & & RV-C vs RV-B $(0.870) \dagger$ \\
\hline Maximal daily symptom score§ & & & & & $0.426 t$ & RV-A vs RV-B (0.216) $\dagger$ \\
\hline No of cases & 200 & 31 & 62 & & & RV-A vs RV-C (0.519) \\
\hline Median (IQR) & $2.3(1.6-3.1)$ & $2.0(1.3-3.1)$ & $2.2(1.5-3.0)$ & & & RV-C vs RV-B (0.499)† \\
\hline Duration of higher symptom score & & & & & $0.942 \dagger$ & RV-A vs RV-B (0.985) $\dagger$ \\
\hline No of cases & 158 & 23 & 48 & & & RV-A vs RV-C (0.740) \\
\hline Median (IQR) days & $4(3-7)$ & $4(2-7)$ & $3(2-6)$ & & & RV-C vs RV-B (0.842) $\dagger$ \\
\hline
\end{tabular}

${ }^{*}$ Characteristics related to disease severity and duration of illness were evaluated only for patients with rhinovirus single infections who had complete case report forms or/and symptom diaries.

tCorrected for age, sex, sample season and sample year.

ҒInitial symptom score scale: $1=$ no problem, $2=$ mild problem, $3=$ moderate problem, $4=$ severe problem.

§Maximal daily symptom score scale: $0=$ no problem/not affected, $1=$ very little problem, $2=$ slight problem, $3=$ moderately bad, $4=$ bad, $5=$ very bad, $6=$ as bad as it could be

$\mathrm{Cq}$, cycle of quantification; RR, relative risk; RV, rhinovirus.

\section{RV type identification}

Of the 686 sequenced RVs, 654 (95\%) were characterised by partial VP3/VP1 or/and VP4/VP2 sequencing and 32 (5\%) based only on the 5'-UTR (see online supplementary appendix). The latter were assigned only to species taxa level, because of limited phylogenetic discrimination in the 5'-UTR region. Four VP4/ VP2 sequences were classified only to species level based on BLASTn results due to poor sequence quality. A total of 650 RV sequences were assigned to 73 RV-A, 25 RV-B and 42 RV-C types representing $91 \%, 78 \%$ and $76 \%,{ }^{11}$ respectively, of the currently established types within each species (figure 3). Five new RV types were identified and assigned by the Picornaviridae study group as RV-A type A109, RV-B types B105 and B106 and RV-C types C52 and C53.

\section{DISCUSSION}

In our study, RV was detected in 19\% of adults with acute cough/ LRTI and in 4\% of follow-up samples as well as samples from asymptomatic controls. The overall annual prevalence ranged from $1 \%$ to $21 \%$ between and within communities. These data indicate that the burden of RV associated illness varies substantially within and between communities in different years. Several other studies have reported rates of RV infection from $7 \%$ to $33 \%$ in inpatient and outpatient adults with acute LRTI or 
Table 3 Frequency and severity of symptoms in adults with rhinovirus associated acute cough/LRTI

\begin{tabular}{|c|c|c|c|c|}
\hline \multirow[b]{2}{*}{ Characteristics } & \multicolumn{3}{|c|}{ No (\%) of events (absent-mild/moderate-severe)* } & \multirow[b]{2}{*}{ P valuet } \\
\hline & $\begin{array}{l}R V-A \\
n=261\end{array}$ & $\begin{array}{l}\text { RV-B } \\
n=43\end{array}$ & $\begin{array}{l}\text { RV-C } \\
n=80\end{array}$ & \\
\hline \multirow[t]{3}{*}{ Cough } & $1(0.4) / 260(99.6)$ & $1(2) / 42(98)$ & $1(1) / 79(99)$ & RV-A vs RV-B (0.053) \\
\hline & & & & RV-A vs RV-C (0.400) \\
\hline & & & & RV-B vs RV-C (0.180) \\
\hline \multirow[t]{3}{*}{ Phlegm } & $131(50) / 130(50)$ & $22(51) / 21(49)$ & $48(60) / 32(40)$ & RV-A vs RV-B (0.739) \\
\hline & & & & RV-A vs RV-C (0.091) \\
\hline & & & & RV-B vs RV-C (0.397) \\
\hline \multirow[t]{3}{*}{ Shortness of breath } & $171(66) / 90(34)$ & $27(63) / 16(37)$ & $55(69) / 25(31)$ & RV-A vs RV-B (0.549) \\
\hline & & & & RV-A vs RV-C (0.578) \\
\hline & & & & RV-B vs RV-C $(0.371)$ \\
\hline \multirow[t]{3}{*}{ Wheeze } & $199(76) / 62(24)$ & $32(74) / 11(26)$ & $64(80) / 16(20)$ & RV-A vs RV-B (0.798) \\
\hline & & & & RV-A vs RV-C (0.606) \\
\hline & & & & RV-B vs RV-C (0.566) \\
\hline \multirow[t]{3}{*}{ Running nose } & $109(42) / 152(58)$ & $21(49) / 22(51)$ & $37(46) / 43(54)$ & RV-A vs RV-B (0.306) \\
\hline & & & & RV-A vs RV-C (0.667) \\
\hline & & & & RV-B vs RV-C (0.545) \\
\hline \multirow[t]{3}{*}{ Fever } & $226(87) / 35(13)$ & $38(88) / 5(12)$ & $69(86) / 11(14)$ & RV-A vs RV-B (0.958) \\
\hline & & & & RV-A vs RV-C (0.883) \\
\hline & & & & RV-B vs RV-C $(0.962)$ \\
\hline \multirow[t]{3}{*}{ Chest pain } & $205(79) / 56(21)$ & $32(74) / 11(26)$ & $60(75) / 20(25)$ & RV-A vs RV-B (0.352) \\
\hline & & & & RV-A vs RV-C (0.534) \\
\hline & & & & RV-B vs RV-C $(0.690)$ \\
\hline \multirow[t]{3}{*}{ Muscle aching } & $189(72) / 72(28)$ & $32(74) / 11(26)$ & $58(73) / 22(27)$ & RV-A vs RV-B (0.662) \\
\hline & & & & RV-A vs RV-C (0.990) \\
\hline & & & & RV-B vs RV-C (0.708) \\
\hline \multirow[t]{3}{*}{ Headache } & $181(69) / 80(31)$ & $25(58) / 18(42)$ & $55(69) / 25(31)$ & RV-A vs RV-B (0.287) \\
\hline & & & & RV-A vs RV-C (0.996) \\
\hline & & & & RV-B vs RV-C $(0.361)$ \\
\hline \multirow[t]{3}{*}{ Disturbed sleep } & $141(54) / 120(46)$ & $25(58) / 18(42)$ & $38(48) / 42(52)$ & RV-A vs RV-B (0.428) \\
\hline & & & & RV-A vs RV-C (0.259) \\
\hline & & & & RV-B vs RV-C (0.150) \\
\hline \multirow[t]{3}{*}{ Diarrhoea } & $256(98) / 5(2)$ & $41(95) / 2(5)$ & $78(98) / 2(2)$ & RV-A vs RV-B (0.087) \\
\hline & & & & RV-A vs RV-C (0.744) \\
\hline & & & & RV-B vs RV-C $(0.225)$ \\
\hline \multirow[t]{3}{*}{ Confusion/ disorientation } & $257(98) / 4(2)$ & $43(100) / 0$ & $79(99) / 1(1)$ & RV-A vs RV-B (0.999) \\
\hline & & & & RV-A vs RV-C (0.999) \\
\hline & & & & RV-B vs RV-C (0.999) \\
\hline \multirow[t]{3}{*}{ Interference with normal activities } & $156(60) / 105(40)$ & $23(53) / 20(47)$ & $54(68) / 26(33)$ & RV-A vs RV-B (0.364) \\
\hline & & & & RV-A vs RV-C (0.281) \\
\hline & & & & RV-B vs RV-C $(0.130)$ \\
\hline \multirow[t]{3}{*}{ Feeling generally unwell } & $118(45) / 143(55)$ & $22(51) / 21(49)$ & $49(61) / 31(39)$ & RV-A vs RV-B (0.442) \\
\hline & & & & RV-A vs RV-C (0.023) \\
\hline & & & & RV-B vs RV-C (0.392) \\
\hline
\end{tabular}

\footnotetext{
*The presence and severity of symptoms were evaluated only for patients with rhinovirus single infections.

tCorrected for age, sex, sample season and sample year.

LRTI, lower respiratory tract infection; RV, rhinovirus.
}

influenza-like illness, ${ }^{152-25}$ and in $8 \%$ or less of asymptomatic adults. $^{3} 41526$

RV species were identified with a different frequency among symptomatic and control subjects. With $68 \%$ prevalence RV-A strains were significantly more common in RV infected symptomatic patients than RV-B (12\%) or RV-C (20\%) strains. In contrast, the prevalence of RV-A (37\%), RV-B (34\%) and RV-C (29\%) was more or less similar in asymptomatic infections, suggesting a more pathogenic role of RV-A infections. Also, RV-A was 4.5 and 2.2 times more often associated with LRTI 

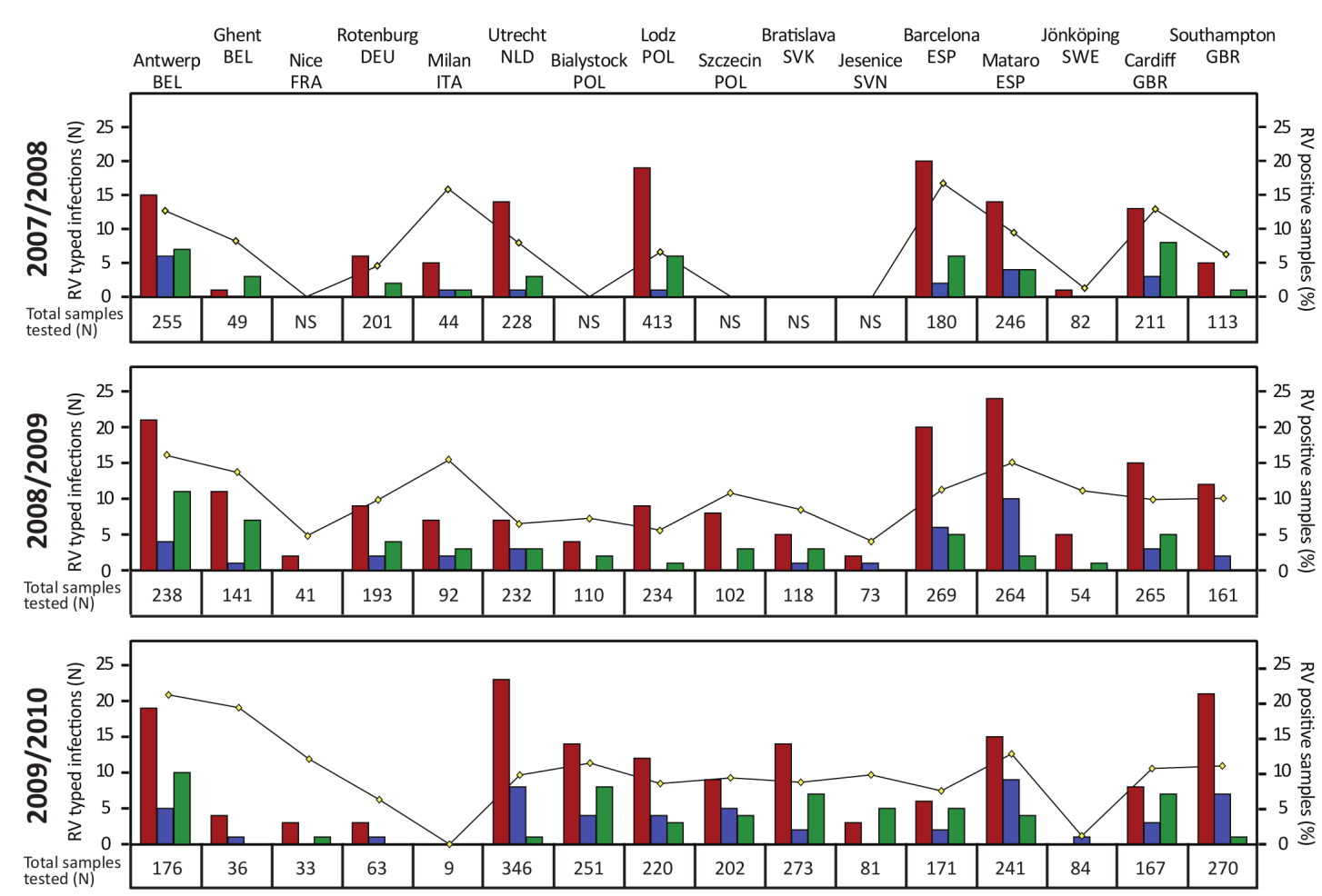

Figure 2 Rhinovirus (RV) detection rate and species distribution according to site and year of detection. Total numbers of tested samples per location and year of isolation are presented under the corresponding country panel; ' $N S^{\prime}$ designates that no samples were available. Plot lines represent the proportion (\%) of RV infections (right side y-axis) and bar graphs represent the number of RV-A (red), RV-B (blue) and RV-C (green) infections (left side y-axis).Country abbreviations: BEL (Belgium), FRA (France), DEU (Germany), ITA (Italy),NLD (Netherland), POL (Poland), SVK (Slovakia), SVN (Slovenia), ESP (Spain),SWE (Sweden), GBR (United Kingdom).

than RV-B and RV-C $(\mathrm{p} \leq 0.010)$, and RV-C 2.1 times more often than RV-B $(p=0.015)$.

Our findings indicate that in adults, the prevalence of RV-Aassociated LRTI is considerably higher than that of RV-B and RV-C, while RV-B infections are more often asymptomatic. The latter observation is further supported by the fact that RV-B has been detected infrequently in children and adults hospitalised for severe respiratory illness. ${ }^{17}{ }^{17-31}$ Furthermore RV-B symptomatic infections had significantly lower viral loads than RV-A symptomatic infections, suggesting lower pathogenicity of RV-B viruses. Lower viral loads of RV-B relative to RV-A and RV-C in respiratory specimens have previously been reported for adults with pneumonia ${ }^{32}$ and viral load has been reported as indicator for severity of RV infections. ${ }^{3}$ These findings are in agreement with the in vitro study that demonstrated that RV-B types replicate less efficiently and induce lower cytokines and/or chemokine levels than RV-A or RV-C infections. ${ }^{33}$

Our results on RV species distribution (RV-A $>$ RVC $>$ RVB) among adults with LRTI are consistent with those from a study conducted in Seoul among adults with pneumonia. ${ }^{32}$ Other studies in symptomatic patients found a lower prevalence of RV-C than RV-B infections. ${ }^{34}{ }^{35}$ These differences in the relative proportions of RV species in comparison to our findings could be due to differences in the study populations (eg, upper respiratory tract infection (URTI) instead of LRTI) and annual variations in species distribution. We observed no significant differences between RV species in the duration of illness, maximum symptom scores, overall disease severity and severity of individual common cold symptoms. However, RV-A infected patients subjectively felt in general worse than those infected with RV-C. Our results suggest that RV-A infections have a more debilitating impact on the general health as compared with RV-B and RV-C infections. In children several studies demonstrated an association between RV-C and more serious respiratory illness. ${ }^{1322} 28$ In adult patients RV-C infection has been reported to cause more frequent acute RTI and appeared more severe as compared with children. ${ }^{22}{ }^{36}$ Other studies in adults observed no differences in disease severity between RV species, ${ }^{15} 16263237$ though one study found a higher disease severity associated with RV-A in upper respiratory symptoms. ${ }^{35}$ However, these studies are limited due to the short surveillance periods, lack of asymptomatic controls and small sample size. It must be noted that the patients included in this study had per definition acute cough/ LRTI, thus differences in disease severity between RV species in patients with milder symptoms cannot be excluded.

RV-A was the most prevalent species in the majority of study locations, followed by RV-C, while RV-B occurrence varied considerably between and within certain communities. Substantial antigenic variation within RV-A could lead to more frequent reinfections in the community and to a higher overall prevalence. Interestingly, not all RV species were identified in the separate locations during the study period. Genotyping of the RV samples lead to the discovery of 5 novel RV types and revealed a remarkably high diversity of RVs represented by 73 RV-A, 25 RV-B and 42 RV-C types. Asymptomatic infections were associated with a variety of $\mathrm{RV}$ types, the majority of which were also identified in symptomatic cases.

Although comprehensive, this study has some limitations. Due to the heterogeneity of the RV genomes, our RV diagnostic and genotyping assays might have varied in efficacy of amplification, and therefore, be suboptimal for some genotypes. In particular, RV-C infections might be somewhat underrepresented, since a limited number of RV-C complete genome sequences were available at the time our diagnostic assay was developed. 

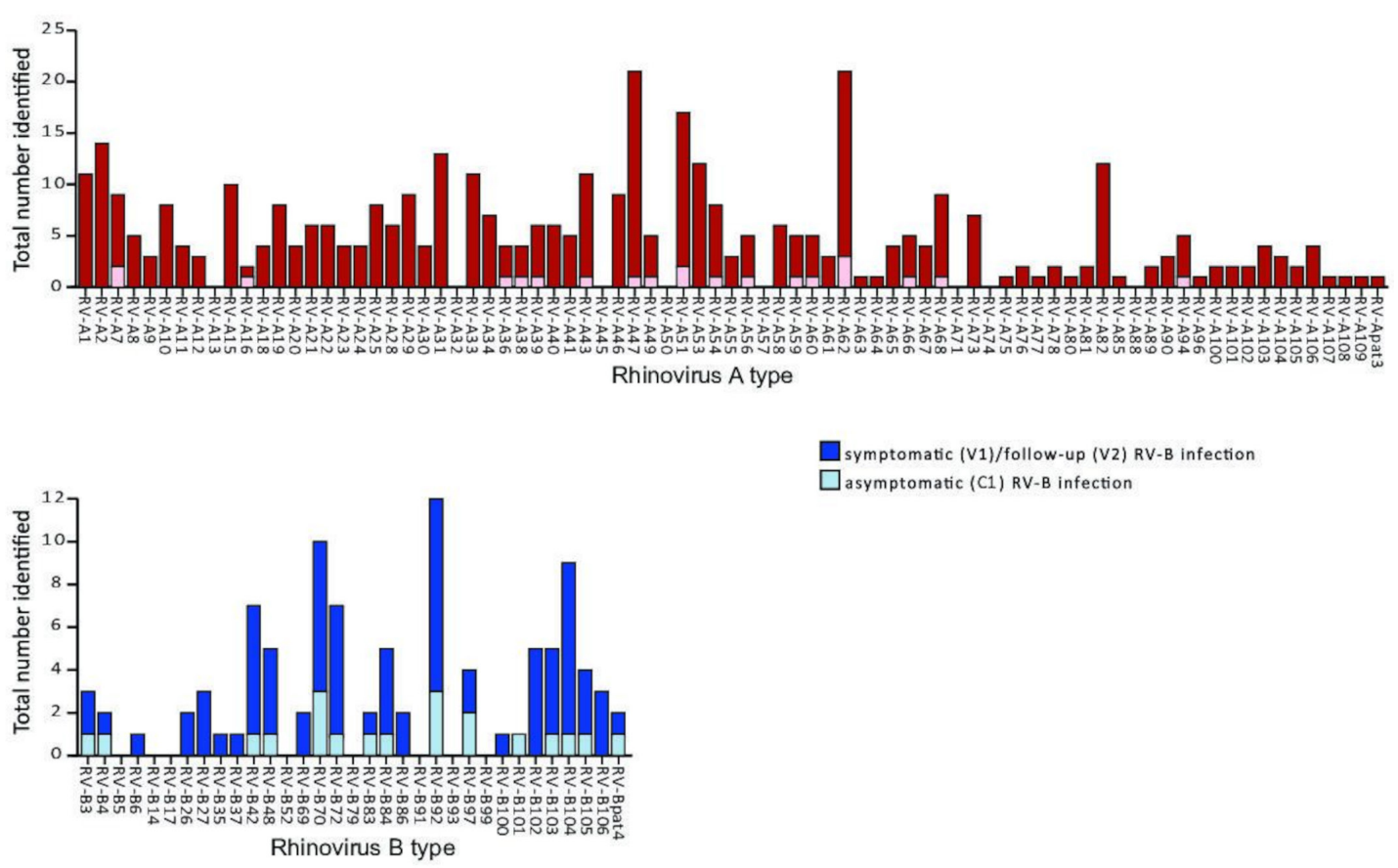

$\square$ symptomatic (V1)/follow-up (V2) RV-B infection

$\square$ asymptomatic (C1) RV-B infection

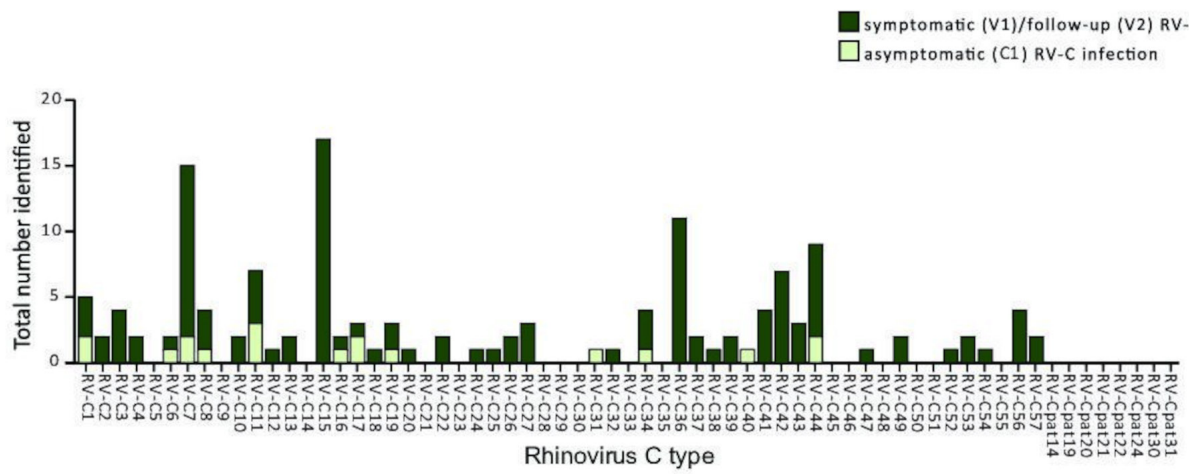

Figure 3 Frequencies of rhinovirus types identified in symptomatic case and asymptomatic control adults.

In addition, due to the nature of this study, only associations can be found, and no causality can be proven. With the current study design, an association can be made between RV type and disease severity. However, the population studied, is patients visiting their GP that in general will be older and have more comorbidities as compared with the general population. Nevertheless, these are the patients consulting their GP, and therefore, this study is very representative for clinical practice.

Although limited, some CRF's were missing and not all RV positive samples could be typed. Because these numbers are limited a link between the missing data and disease severity is not expected and this probably will not result in a bias.

Finally, the clinical status of cases with RV-positive follow-up samples could not be clearly defined, since symptom manifestations were not recorded by the GP at the time of the second visit.

This is a very comprehensive study, in which RV typing has been performed in symptomatic (LRTI) and asymptomatic adult GP patients in 11 countries. RV typing is not part of standard diagnostic care and our results do not provide an indication for an added value in relation to disease severity. This may change in the future if subtype differences in efficacy are observed following antiviral treatment or vaccination.

In conclusion, LRTI in adults were mainly associated with $\mathrm{RV}-\mathrm{A}$, identified in a significantly higher proportion of symptomatic than asymptomatic subjects as compared with RV-B and RV-C. Furthermore RV-A symptomatic infections were associated with significantly higher viral loads than RV-B symptomatic infections, suggesting lower pathogenicity of RV-B.

\section{Author affiliations}

${ }^{1}$ Medical Microbiology, Leiden Universitair Medisch Centrum, Leiden, The Netherlands

${ }^{2}$ Infection and Immunity Division, University of Edinburgh, Edinburgh, UK

${ }^{3}$ Department of Medical Microbiology, University Medical Center Utrecht, Utrecht, The Netherlands

${ }^{4}$ Department of Data Management, Julius Center for Health Sciences and Primary Care, University Medical Center Utrecht, Utrecht, The Netherlands

${ }^{5}$ Primary Care and Population Science, University of Southampton, Southampton, UK

${ }^{6}$ Nuffield Department of Primary Care Sciences, Oxford University, Oxford, UK

${ }^{7}$ Department of Medical Statistics, Leiden University Medical Center, Leiden, The Netherlands

${ }^{8}$ Department of Medical Microbiology, Vaccine \& Infectious Diseases Institute (VAXINFECTIO), University of Antwerp, Antwerp, Belgium 
Acknowledgements We thank all involved in the GRACE Primary Care Network for the opportunity to conduct this study, Stefan van Nieuwkoop, Naim Farzad, Yuvia Guilarte, Iris Hidding, Lisette Rusman, Margriet Kraakman and Mario van Bussel (Department of Medical Microbiology, LUMC, Leiden, The Netherlands) for technical assistance with virus genotyping, and Susan Hemert-Glaubitz (Julius Center of Health Sciences and Primary Care, UMC, Utrecht, The Netherlands) for assistance with the GRACE database.

Funding This study was funded by the of the European Commision's FP6 Programme under grant number LSHM-CT-2005-5 18226 and the Department of Medical Microbiology, Leiden University Medical Centre, the Netherlands.

Competing interests None declared.

Patient consent for publication Not required.

Provenance and peer review Not commissioned; externally peer reviewed.

Data availability statement Data are available on reasonable request. RV sequences from this study have been deposited in GenBank under the accession numbers KP736530-KP737279 and KR045604.

Open access This is an open access article distributed in accordance with the Creative Commons Attribution Non Commercial (CC BY-NC 4.0) license, which permits others to distribute, remix, adapt, build upon this work non-commercially, and license their derivative works on different terms, provided the original work is properly cited, appropriate credit is given, any changes made indicated, and the use is non-commercial. See: http://creativecommons.org/licenses/by-nc/4.0/.

ORCID iDs

Anneloes L van Rijin http://orcid.org/0000-0002-0551-5543

Paul Little http://orcid.org/0000-0003-3664-1873

\section{REFERENCES}

1 Heikkinen T, Järvinen A. The common cold. Lancet 2003;361:51-9.

2 van Gageldonk-Lafeber AB, Heijnen M-LA, Bartelds AIM, et al. A case-control study of acute respiratory tract infection in general practice patients in the Netherlands. Clin Infect Dis 2005;41:490-7.

$3 \mathrm{Ng} \mathrm{KT}$, Oong XY, Lim SH, et al. Viral load and sequence analysis reveal the symptom severity, diversity, and transmission clusters of rhinovirus infections. Clin Infect Dis 2018;67:261-8.

4 van der Linden L, Bruning AHL, Thomas XV, et al. A molecular epidemiological perspective of rhinovirus types circulating in Amsterdam from 2007 to 2012. Clin Microbiol Infect 2016;22:1002.e9-14.

5 Ruuskanen 0 , Waris M, Ramilo 0 . New aspects on human rhinovirus infections. Pediatr Infect Dis J 2013;32:553-5.

6 Vandini S, Biagi C, Fischer M, et al. Impact of rhinovirus infections in children. Viruses 2019;11:521.

7 Goffard A, Lambert V, Salleron J, et al. Virus and cystic fibrosis: rhinoviruses are associated with exacerbations in adult patients. J Clin Virol 2014;60:147-53.

8 Wu X, Chen D, Gu X, et al. Prevalence and risk of viral infection in patients with acute exacerbation of chronic obstructive pulmonary disease: a meta-analysis. Mol Biol Rep 2014;41:4743-51.

9 Goka EA, Vallely PJ, Mutton KJ, et al. Single, dual and multiple respiratory virus infections and risk of hospitalization and mortality. Epidemiol Infect 2015;143:1-11.

10 Honkinen $\mathrm{M}$, Lahti E, Österback R, et al. Viruses and bacteria in sputum samples of children with community-acquired pneumonia. Clin Microbiol Infect 2012;18:300-7.

11 ICTV. Genus: enterovirus. Available: https://talk.ictvonline.org/ictv-reports/ictv_ online_report/positive-sense-rna-viruses/picornavirales/w/picornaviridae/681/genusenterovirus [Accessed 25 Jul 2019].

12 Lau SKP, Yip CCY, Tsoi H-W, et al. Clinical features and complete genome characterization of a distinct human rhinovirus (HRV) genetic cluster, probably representing a previously undetected HRV species, HRV-C, associated with acute respiratory illness in children. J Clin Microbiol 2007;45:3655-64.

13 Piralla A, Rovida F, Campanini G, et al. Clinical severity and molecular typing of human rhinovirus C strains during a fall outbreak affecting hospitalized patients. J Clin Virol 2009;45:311-7.
14 Hasegawa K, Mansbach JM, Bochkov YA, et al. Association of rhinovirus C bronchiolitis and immunoglobulin E sensitization during infancy with development of recurrent wheeze. JAMA Pediatr 2019;173:544.

15 Fry AM, Lu X, Olsen SJ, et al. Human rhinovirus infections in rural Thailand: epidemiological evidence for rhinovirus as both pathogen and bystander. PLoS One 2011;6:e17780.

16 Henquell C, Mirand A, Deusebis A-L, et al. Prospective genotyping of human rhinoviruses in children and adults during the winter of 2009-2010. J Clin Virol 2012;53:280-4.

17 Iwane MK, Prill MM, Lu X, et al. Human rhinovirus species associated with hospitalizations for acute respiratory illness in young US children. J Infect Dis 2011;204:1702-10.

18 Ren L, Yang D, Ren X, et al. Genotyping of human rhinovirus in adult patients with acute respiratory infections identified predominant infections of genotype A21. Sci Rep 2017;7:41601.

19 leven M, Coenen S, Loens K, et al. Aetiology of lower respiratory tract infection in adults in primary care: a prospective study in 11 European countries. Clin Microbiol Infect 2018;24:1158-63.

20 Zlateva KT, de Vries JJC, Coenjaerts FEJ, et al. Prolonged shedding of rhinovirus and re-infection in adults with respiratory tract illness. Eur Respir J 2014;44:169-77.

21 Loens K, van Loon AM, Coenjaerts F, et al. Performance of different mono- and multiplex nucleic acid amplification tests on a multipathogen external quality assessment panel. J Clin Microbiol 2012;50:977-87.

22 Lau SKP, Yip CCY, Lin AWC, et al. Clinical and molecular epidemiology of human rhinovirus $C$ in children and adults in Hong Kong reveals a possible distinct human rhinovirus C subgroup. J Infect Dis 2009;200:1096-103.

23 Templeton KE, Scheltinga SA, van den Eeden WCJFM, et al. Improved diagnosis of the etiology of community-acquired pneumonia with real-time polymerase chain reaction. Clin Infect Dis 2005;41:345-51.

24 Pierangeli A, Scagnolari C, Selvaggi C, et al. Rhinovirus frequently detected in elderly adults attending an emergency department. J Med Virol 2011;83:2043-7.

25 Creer DD, Dilworth JP, Gillespie SH, et al. Aetiological role of viral and bacterial infections in acute adult lower respiratory tract infection (LRTI) in primary care. Thorax 2006;61:75-9.

26 Granados A, Goodall EC, Luinstra K, et al. Comparison of asymptomatic and symptomatic rhinovirus infections in university students: incidence, species diversity, and viral load. Diagn Microbiol Infect Dis 2015;82:292-6.

27 Lee W-M, Lemanske RF, Evans MD, et al. Human rhinovirus species and season of infection determine illness severity. Am J Respir Crit Care Med 2012;186:886-91.

28 Linsuwanon P, Payungporn S, Samransamruajkit R, et al. High prevalence of human rhinovirus $C$ infection in Thai children with acute lower respiratory tract disease. J Infect 2009;59:115-21.

29 Miller EK, Khuri-Bulos N, Williams JV, et al. Human rhinovirus C associated with wheezing in hospitalised children in the middle East. J Clin Virol 2009;46:85-9.

30 Hung H-M, Yang S-L, Chen C-J, et al. Molecular epidemiology and clinical features of rhinovirus infections among hospitalized patients in a medical center in Taiwan. $J$ Microbiol Immunol Infect 2019;52:233-41.

31 Liggett SB, Bochkov YA, Pappas T, et al. Genome sequences of rhinovirus $C$ isolates from Wisconsin pediatric respiratory studies. Genome Announc 2014;2. doi:10.1128/ genomeA.00203-14. [Epub ahead of print: 27 Mar 2014].

32 Choi S-H, Hong S-B, Kim T, et al. Clinical and molecular characterization of rhinoviruses a, B, and C in adult patients with pneumonia. J Clin Virol 2015;63:70-5.

33 Nakagome K, Bochkov YA, Ashraf S, et al. Effects of rhinovirus species on viral replication and cytokine production. J Allergy Clin Immunol 2014;134:332-41.

34 Xiang Z, Gonzalez R, Wang Z, et al. Human rhinoviruses in Chinese adults with acute respiratory tract infection. J Infect 2010;61:289-98.

35 Chen W-J, Arnold JC, Fairchok MP, et al. Epidemiologic, clinical, and virologic characteristics of human rhinovirus infection among otherwise healthy children and adults: rhinovirus among adults and children. J Clin Virol 2015:64:74-82.

36 Wisdom A, Leitch ECM, Gaunt E, et al. Screening respiratory samples for detection of human rhinoviruses (HRVs) and enteroviruses: comprehensive VP4-VP2 typing reveals high incidence and genetic diversity of HRV species C. J Clin Microbiol 2009;47:3958-67.

37 Pretorius MA, Tempia S, Treurnicht FK, et al. Genetic diversity and molecular epidemiology of human rhinoviruses in South Africa. Influenza Other Respir Viruses 2014;8:567-73. 